Gesnerus 52 (1995) 372-376

\title{
Contents of Vol. 52 (1995)
}

\section{Original Articles}

Bellwinkel, Hans Wolfgang: Dürrenmatt und die Naturwissenschaften [Dürrenmatt and

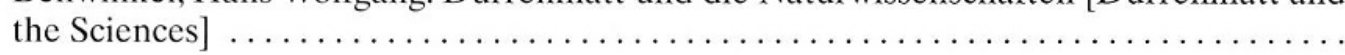

Borch-Jacobsen, Mickel; Shamdasani, Sonu:Ein Dokument:Das Problem der Willensfreiheit und die Psychoanalyse. Eine psychoanalytische Schrift des «Wolfsmanns» [A Document:The Problem of Free Will in Psychanalysis. A Psychanalytic Text of the Wolfman]

Greif, Rudolf; Schmutz, Hans-Konrad: Hans Bluntschli als Morphologe [Hans Bluntschli,

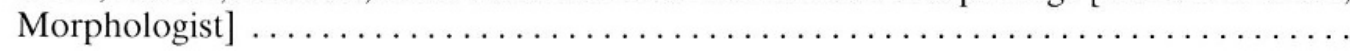

Grmek, Mirko D.: Les médecins communaux de Raguse (Dubrovnik) au Moyen Age [The Communal Physicians of Ragusa (Dubrovnik) in the Middle Ages] ................

Hirschmüller, Albrecht: E. Merck und das Kokain. Zu Sigmund Freuds Kokainstudien und ihren Beziehungen zu der Darmstädter Firma [E. Merck and Cocaine. Freud's Cocaine Studies and their Connection with the Merck Company in Darmstadt] .......

McNiven Hine, Ellen: Dortous de Mairan and Eighteenth Century "Systems Theory" ..

Neiger, Markus; Boschung, Urs: Die Behandlung Ohrenkranker in Bern in der ersten Hälfte des 19. Jahrhunderts [The Treatment of Ear Patients in Bern During the First Half

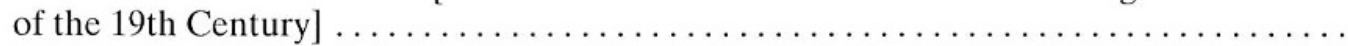

Nicoli Aldini, Nicolò: The First Billroth II Gastric Resection as Reported by an Italian Medical Journal

Prüll, Cay-Rüdiger: Die Grundkonzepte der Pathologie in Deutschland von 1858 bis heute und der Fortschrittsbegriff in der Medizin [Basic Concepts in German Pathology

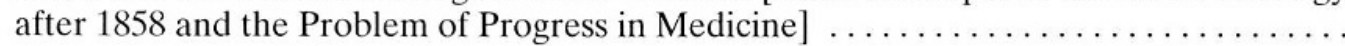

Raggenbass, René: La fièvre bilieuse hémoglobinurique: un épisode français tiré d'une recherche d'Alexandre Yersin [Blackwater Fever: A French Episode Based on an Inves-

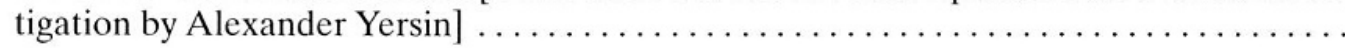

Schnalke, Thomas: Praxis, Theorie und Wissenschaft im Selbstverständnis des städtischen Arztes Christoph Jacob Trew (1695-1769) [Practice, Theory, and Sciences as Seen by the Urban Practitioner, Christoph Jacob Trew (1695-1769)] . . . . . . . . . . . . . . . .

Schulte-Bockholt, Arnd; Bauer, Axel: Innere Medizin in den deutschsprachigen Ländern und in den USA. Wechselseitige Einflüsse und Wandel der Beziehungen von 1870 bis 1990 [Internal Medicine in the German-speaking Countries and in the U.S.A. Mutual Influences and Changing Relationships from 1870 to 1990$] \ldots \ldots \ldots \ldots \ldots \ldots \ldots \ldots \ldots \ldots$ 
Steinmann, Mathias: Impfalltag im 19. Jahrhundert. Das Verhältnis zwischen Ärzten und Bevölkerung vor dem Hintergrund der Pockenschutzimpfung im Kanton Luzern [Vacci-

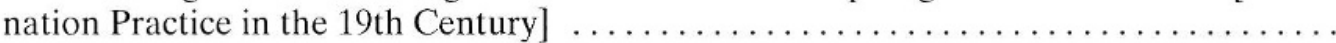

Weiner, Dora B.: Les femmes de la Salpêtrière: trois siècles d'histoire hospitalière parisienne [The Women of the Salpêtrière: three Centuries of History of Hospitals in Paris]

\section{Editorials}

Barras, Vincent: Pour l'anniversaire de Jean Starobinski. Bibliographie des travaux de Jean Starobinski en histoire de la médecine [Jean Starobinski, Anniversary and Bibliography of his Medico-historical Works] . . . . . . . . . . . . . . . . . . . . . .

Bickel, Marcel H.: Gesnerus neu im Verlag Schwabe, Basel .................... 5

\section{Short Communications}

Daxecker, Franz; Broucek, Annemarie: Eine Darstellung der hl. Ottilie mit Lesesteinen [A Representation of Saint Ottilia with Reading Stones] ....................

Mörgeli, Christoph:Wilhelm Conrad Roentgen and Switzerland.Exhibition in the Museum of the History of Medicine of the University of Zurich, May 18th to December 17th, 1995

Obituaries

News and Activities

\section{Book Reviews}

Actas del IX Congreso Nacional de Historia de la Medicina, Zaragoza, 1989 (Barras) . . 169

Aird, Robert B.: Foundations of modern neurology (Isler)

Albrecht, Michael: Eklektik. Eine Begriffsgeschichte mit Hinweisen auf die Philosophie-

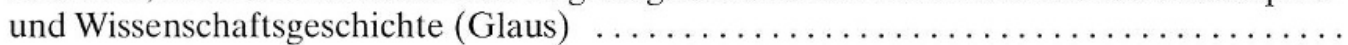

Althoff, Jochen: Warm, kalt, flüssig und fest bei Aristoteles (Orelli Facchini) . . . . . . . 34

Anglin, W. S.: Mathematics: a concise history and philosophy (Burckhardt) $\ldots \ldots \ldots \ldots$

Aristoteles. Werke. Band 14: Parva naturalia. Teil 3: De insomniis, De divinatione per somnum. Übers. und erläutert von Philip J. van der Eijk (Näf) $\ldots \ldots \ldots \ldots \ldots \ldots \ldots$

Barona Vilar, Josep Lluis: La doctrina y el laboratorio. Fisiología y experimentación en la sociedad española del siglo XIX (Keller-Schnider) $\ldots \ldots \ldots \ldots \ldots \ldots \ldots \ldots \ldots \ldots \ldots$

Bernabeu Mestre, Josep; Olagüe de Ros, Guillermo; Peiró Cabrera, Gloria: Catàleg del fons científic (segles XVI/XVIII) de la biblioteca «Fernando de Loaces», Oriola

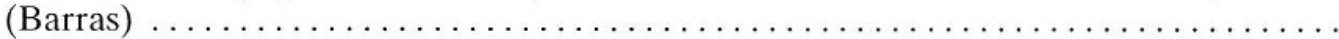

Bleker, Johanna; Jachertz, Norbert (Hrsg.): Medizin im «Dritten Reich» (Nickol) . . . . . 167

Bourbaki, Nicolas: Elements of the history of mathematics (Gantenbein) $\ldots \ldots \ldots \ldots \quad 192$ 
Brossolet, Jacqueline; Mollaret, Henri: Pourquoi la peste? (Kupferschmidt) . . . . . . 358

Bulst, Neithard; Delort, Robert: Maladies et société (XII - XVIII ${ }^{\mathrm{e}}$ siècles) (Seiler) . . . 337

Cadden, Joan: Meanings of sex difference in the Middle Ages (Seiler) …....... 186

Caelius Aurelianus: Akute Krankheiten Buch I-III, Chronische Krankheiten Buch I-V

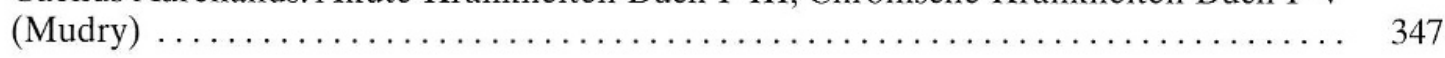

Caneva, Kenneth L.: Robert Mayer and the conservation of energy (Funk) ........ 345

Catalogue de la correspondance de Charles Bonnet (Boschung) . . . . . . . . . . 176

Cliff, Andrew; Haggett, Peter; Smallman-Raynor, Matthew: Measles (Lindenmann) . . . 343

Companion encyclopedia of the history and philosophy of the mathematical sciences

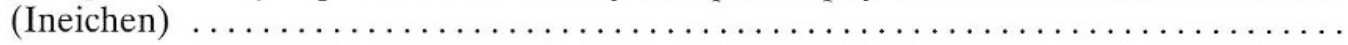

Cordes, Peter: Iatros. Das Bild des Arztes in der griechischen Literatur von Homer bis

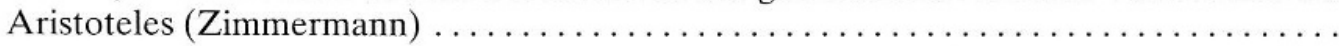

Curing and insuring. Essays on illness in past times. Conference Illness and History,

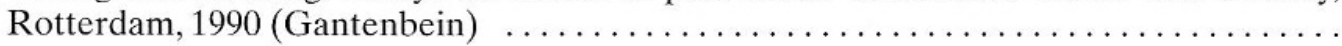

Daal, M. J. G. W. van; Knecht-van Eekelen, A. de: Joannes Juda Groen (1903-1990)

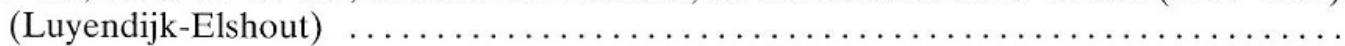

Dugo, Franciscus Prosper: Fons Aquae Bonae. Dass ist Kurtzer Bericht von dem Bad zu Bonn und seiner Würckung ... 1662 (Rudolph) $\ldots \ldots \ldots \ldots \ldots \ldots \ldots \ldots \ldots \ldots \ldots \ldots \ldots \ldots$

Emeriti erinnern sich. Rückblicke auf die Lehre und Forschung in Heidelberg (Bickel) .

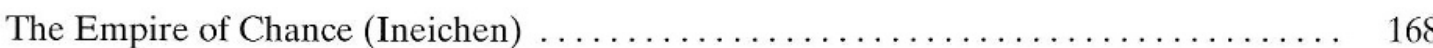

Galenus latinus II: Burgundio of Pisa's translation of Galen's Peri tōn peponthotōn to-

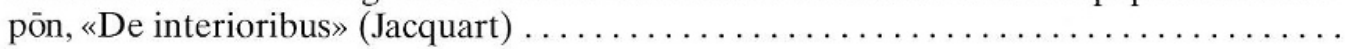

Gebhardt, Rainer: Einblicke in die Coss von Adam Ries (Burckhardt) ........... 172

Grote, Andreas (Hrsg.): Macrocosmos in Microcosmos. Die Welt in der Stube. Zur Geschichte des Sammelns 1450 bis 1800 (Hagner) .........................

Guitton, Jean; Bogdanov, Grichka und Igor: Gott und die Wissenschaft (Illhardt) . . . . 166

Hirschmüller, Albrecht: Freuds Begegnung mit der Psychiatrie: von der Hirnmythologie

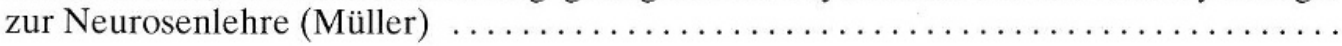

Horstmanshoff, H. F. J. (ed.): Pijn en balsem, troost en smart (Luyendijk-Elshout) . ....

Houtzager, H. L.: Wat er in de kraam te pas komt (Müller-Landgraf) . . . . . . . . . . 347

Huisman, Frank: Stadsbelang en Standsbesef (Müller-Landgraf) . . . . . . . . . . . . 340

Imbert, Jean: Le droit hospitalier de l'Ancien Régime (Mayer) $\ldots \ldots \ldots \ldots \ldots \ldots \ldots$

Imhof, Arthur E.: Lebenserwartungen in Deutschland vom 17. bis 19. Jahrhundert; id. (Hrsg.): Lebenserwartungen in Deutschland, Norwegen und Schweden im 19. und

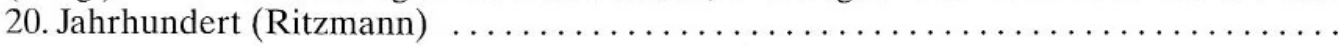

Jacyna, L. S. (ed.): A tale of three cities: the correspondence of William Sharpey and Allen Thomson (Steiner) 
Jagella, Caroline; Isler, Hansruedi; Hess, Klaus: 100 Jahre Neurologie an der Universität Zürich 1894 bis 1994. - Constantin von Monakow (1853 bis 1930), Hirnforscher, Neuro-

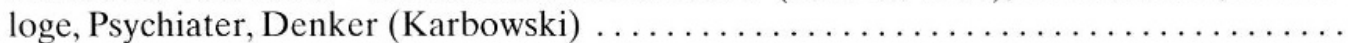

Jahn, Ellen: Die Cholera in Medizin und Pharmazie im Zeitalter des Hygienikers Max

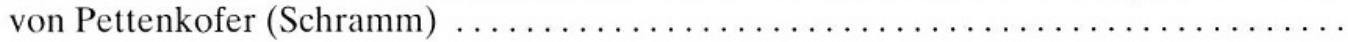

Juskevic, Adolf P.; Kopelevic, Judith Kh.: Christian Goldbach 1690-1764 (Burckhardt) .

Kanz, Kai Torsten (Hrsg.): Philosophie des Organischen in der Goethezeit: Studien zu Werk und Wirkung des Naturforschers Carl Friedrich Kielmeyer (1765-1844) (Ziswiler)

Keppel Hesselink, J. M.: Beelden in de mist. De geschiedenis van der neurologie in capita selecta (Isler)

Kössler,Henning (Hrsg.): 250 Jahre Friedrich-Alexander-Universität Erlangen-Nürnberg

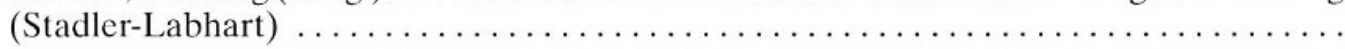

Kupferschmidt, Hugo: Die Epidemiologie der Pest (Wüst) $\ldots \ldots \ldots \ldots \ldots \ldots \ldots \ldots$ 182

Labisch, Alfons: Homo Hygienicus: Gesundheit und Medizin in der Neuzeit (Seiler) . . .

Ledermann, François (Hrsg.): Festschrift zum 150jährigen Bestehen des Schweizerischen

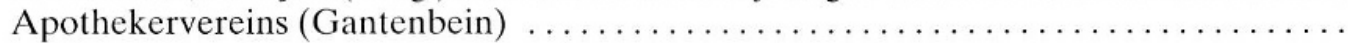

Lieburg, M. J. van: Het Sint Franciscus Gasthuis te Rotterdam 1892-1992; id.: Van Zeemanshospital tot Havenziekenhuis (Müller-Landgraf) $\ldots \ldots \ldots \ldots \ldots \ldots \ldots \ldots$

Lindeboom, G. A.: Inleiding tot de geschiedenis der geneeskunde (Huizink) . . . . . . . 185

Loetz, Francisca: Vom Kranken zum Patienten. «Medikalisierung» und medizinische Vergesellschaftung am Beispiel Badens 1750-1850 (Böni) . . . . . . . . . . . . . . .

Longrigg, James: Greek rational medicine (Mudry) $\ldots \ldots \ldots \ldots \ldots \ldots \ldots \ldots \ldots \ldots$

Mackay, Judith: Der Weltgesundheits-Atlas (Ritzmann) $\ldots \ldots \ldots \ldots \ldots \ldots \ldots \ldots$

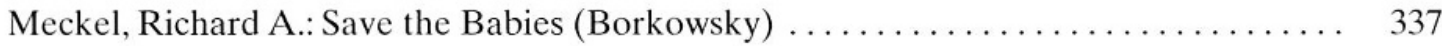

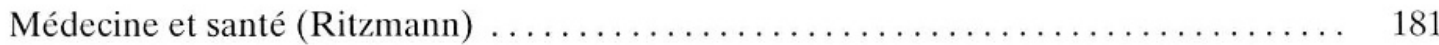

The medical casebook of William Brownrigg, M.D., F.R.S. (1712-1800) of the town of

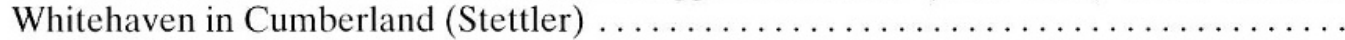

Messengers of mathematics: European mathematical journals (1800-1946) (Neuen-

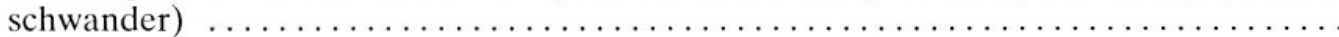

Monti, Maria Teresa: Catalogo del Fondo Haller della Biblioteca Nazionale Braidense di

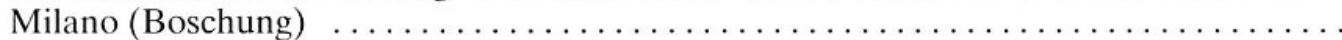

Mosimann, Martin: Die «Mainauer Naturlehre» im Kontext der Wissenschaftsgeschichte

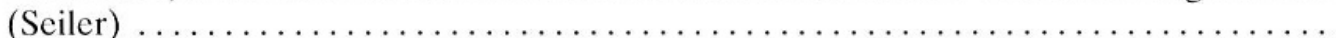

Nach Hadamar. Zum Verhältnis von Psychiatrie und Gesellschaft im 20. Jahrhundert

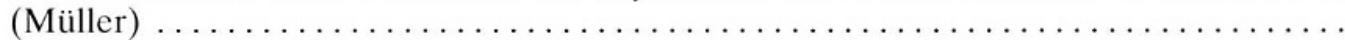

Nater, J. P.: De dood is in de pot, man Gods! (Huizink) $\ldots \ldots \ldots \ldots \ldots \ldots \ldots \ldots$ 184 361

Neuenschwander, Erwin (Hrsg.): Wissenschaft, Gesellschaft und politische Macht

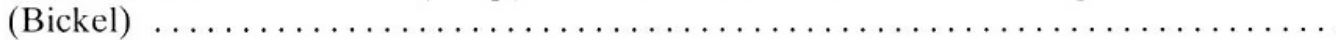

Parthier, Benno: Die Leopoldina (Stadler-Labhart) . . . . . . . . . . . . . . . . 190 
Pfeiffer, Wolfgang M.: Transkulturelle Psychiatrie (Müller)

Pilavas, Ioannis: Psychiatrie im Widerstreit der Konzepte (Müller)

Puenzieux, Dominique; Ruckstuhl, Brigitte: Medizin, Moral und Sexualität (Ritzmann)

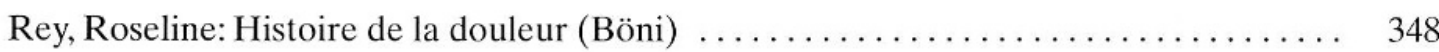

Rheinberger, Hans-Jörg: Experiment - Differenz - Schrift (Schmutz) . . . . . . . . . . 346

Rosarium Philosophorum. Ein alchemisches Florilegium des Spätmittelalters (Gantenbein) 341

Schertenleib, Urban: Kartographie in Winterthur (Brun-Ganzer) 351

Schneider, Ivo: Johannes Faulhaber (1580-1635) (Burckhardt) 179

Schwokowski, Christian (Hrsg.): Friedrich Trendelenburg, 1844-1924 (Naef) . . . . . . . . .

Seidler, Eduard: Die Medizinische Fakultät der Albert-Ludwigs-Universität Freiburg im Breisgau (Koelbing) . . . . . . . . . . . . . . . . . . . . . . . . . . .

Soranus (Ephesius): Soranos d'Ephèse: Maladies des femmes (Mudry) $\ldots . \ldots \ldots \ldots$. 170

Stille, Günther:Der Weg der Arznei von der Materia Medica zur Pharmakologie. Der Weg von Arzneimittelforschung und Arzneitherapie (Ledermann) $\ldots \ldots \ldots \ldots \ldots \ldots$

Stille, Günther: Krankheit und Arznei. Die Geschichte der Medikamente (Ledermann) 366

Stol, Marten: Epilepsy in Babylonia (Gantenbein) . . . . . . . . . . . . . . . . . 177

Valentin, Michel: Louis-René Villermé et son temps (1782-1863) (Gutzwiller) ....... 179

Van der Heyden, J. T. M.: Het ziekenhuis door de eeuwen (Luyendijk-Elshout) . . . . . . 363

Wehefritz, Valentin (Hrsg.): Bibliographie zur Geschichte der Chemie und der chemischen Technologie, 17. bis 19. Jahrhundert (Gantenbein) . . . . . . . . . . . . . . . .

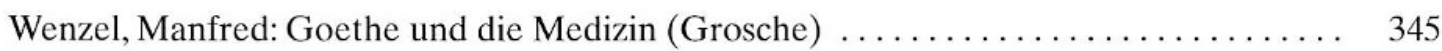

Wenzel, Manfred (Hrsg.): Samuel Thomas Soemmerring in Kassel (1779-1784) (Stadler) 350

Winau, Rolf (Hrsg.): Technik und Medizin (Boschung) . . . . . . . . . . . . . . . 343

Wolfschmidt, Gudrun (Hrsg.): Nicolaus Copernicus (1473-1543). Revolutionär wider

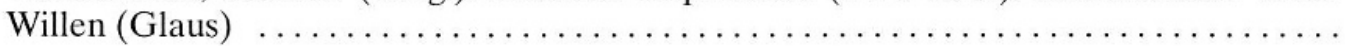

Zerobin, Claudia: Drei Berner Apotheker des 19. Jahrhunderts: Johann Samuel Friedrich Pagenstecher, Carl Abraham Fueter, Leonhard Christian Müller (Fehlmann) .........

Books Received 195,367

Addresses of Reviewers

199,370 